\title{
Gestão financeira de unidades de conservação: perspectiva teórica sobre turismo e uso público em Minas Gerais, Brasil
}

\section{Camila Magri Bertolin}

Universidade Federal de Ouro Preto. Programa de Pós-Graduação em Sustentabilidade Socioeconômica Ambiental. Escola de Minas. Campus Morro do Cruzeiro. Ouro Preto-MG, Brasil (CEP 35400-000). E-mail: camilamagribertolin@gmail.com.

Resumo. A gestão das unidades de conservação muitas vezes pode ser considerada ineficiente devido, dentre outros fatores, à necessidade de recursos financeiros. Em Minas Gerais, essas unidades proporcionam benefícios diretos e indiretos à população, a qual deve possuir condições de acesso equitativo a tais serviços. Este estudo teve como objetivo desenvolver estudo teórico sobre a gestão financeira das unidades de conservação de Minas Gerais, ressaltando aspectos de turismo e uso público. Neste trabalho, de caráter exploratório e descritivo, foram utilizadas como método pesquisa bibliográfica, pesquisa documental e análise de conteúdo. Chegou-se ao entendimento que, diante do cenário de dificuldades relativas a disponibilização de recursos, as unidades devem ser analisadas também sob aspectos gerenciais e alternativas financeiras devem ser criadas. A taxa de visitação é considerada importante fonte de arrecadação, além das parcerias e cobrança pela exploração da prática de esportes dentro das unidades. Os artigos pesquisados reforçam a ideia de uso público nas unidades de conservação e demonstram a importância e pertinência do turismo sustentável. Foram observadas experiências positivas, tanto em Minas Gerais como no Brasil.

Palavras-chave: Áreas protegidas; Sustentabilidade financeira; Visitação.

\begin{abstract}
Financial management of conservation units: Theoretical perspective on tourism and public use in Minas Gerais, Brazil. The management of conservation units many times can be considered inefficient due, among other factors, to the need for financial resources. In Minas Gerais State, these units provide direct and indirect benefits to the population, which must have conditions of equitable access to such services. This paper aimed to develop a theoretical study on financial management of conservation units in Minas Gerais, highlighting aspects of tourism and public use. In this work, of an exploratory and descriptive character, bibliographic research, documentary
\end{abstract}

Recebido

10/02/2020

Aceito

29/04/2020

Publicado

$30 / 04 / 2020$

Acesso aberto

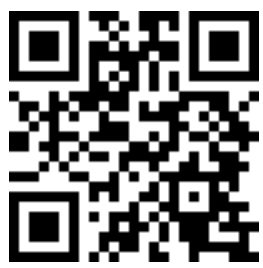

ORCID

(D) 0000-0001-5752-8015 Camila Magri Bertolin

ISSN 2359-1412/RBGAS-2020-0019/2020/7/15/27/373

Rev. Bras. Gest. Amb. Sustent.

http://revista.ecogestaobrasil.net 
research and content analysis were used as methods. It was understood that, given the scenario of difficulties related to the availability of resources, the units must also be analyzed under managerial aspects and financial alternatives must be created. The visitation fee is considered an important source of revenue, in addition to partnerships and charges for the exploration of sports inside the units. The researched articles reinforce the idea of public use in conservation units and demonstrate the importance and pertinence of sustainable tourism. Positive experiences were observed, both in Minas Gerais and in Brazil.

Keywords: Protected areas; Financial sustainability; Visitation.

\section{Introdução}

Os ecossistemas terrestres estão sendo deteriorados cada vez mais pela ação humana. De acordo com Godoy e Leuzinger (2015), a criação de áreas naturais protegidas, dentre elas as unidades de conservação, encontram-se neste contexto como estratégia fundamental relativa à conservação da biodiversidade e preservação da fauna e flora em prol das futuras gerações. Porém, segundo Bensusan e Prates (2014), essas áreas não têm obtido êxito em conter a perda crescente de biodiversidade.

A gestão das áreas protegidas, tanto no Brasil quanto no exterior, pode ser considerada ineficiente. Estas áreas necessitam de mais recursos financeiros a fim de dar continuidade à tarefa da conservação (Quammen, 2016). Na Lei no 9.985/2000, instituidora do Sistema Nacional de Unidades de Conservação da Natureza (SNUC), são previstos a alocação adequada dos recursos financeiros a fim de realizar gestão efetiva das unidades de conservação, assegurando sua sustentabilidade econômica, assim como a promoção do desenvolvimento sustentável a partir dos recursos naturais e a valorização econômica e social da diversidade biológica (Brasil, 2000). Logo, a gestão financeira das unidades de conservação pode ser declarada ferramenta importante no sentido de manutenção e continuidade das mesmas.

Estudos e pesquisas direcionados aos aspectos econômicos das unidades de conservação são escassos, ainda que sejam considerados indispensáveis ao planejamento e ao alcance dos objetivos gerenciais das mesmas (Muanis et al., 2009; Dias, 2013). Portanto, apresenta-se como objetivo geral desenvolver estudo teórico sobre a gestão financeira das unidades de conservação de Minas Gerais, ressaltando aspectos de turismo e uso público. Como objetivos específicos, pretende pesquisar e examinar aspectos da gestão de unidades de conservação de Minas Gerais, especialmente os financeiros. Evidenciar as possibilidades existentes de uso público nas unidades de conservação. Investigar a relevância do uso público em unidades de conservação, focando no turismo, por meio da observação e análise de estudos teóricos de experiências bem-sucedidas.

\section{Gestão de unidades de conservação}

Apontada como conquista e marco na legislação ambiental brasileira, a lei do SNUC contribuiu com avanços nas políticas de ampliação e gestão das áreas naturais protegidas por unidades de conservação em todas as esferas de governo, criando mecanismos que possibilitam a participação da sociedade nessa gestão. Nos últimos anos, ocorreu aumento significativo na área abrangida por unidades de conservação, e pode-se dizer que as inovações trazidas pela lei foram importantes em relação a essa expansão (Sousa et al., 2011). Do ponto de vista legal, as unidades de conservação podem ser definidas como: 
espaço territorial e seus recursos ambientais, incluindo as águas jurisdicionais, com características naturais relevantes, legalmente instituído pelo Poder Público, com objetivos de conservação e limites definidos, sob regime especial de administração, ao qual se aplicam garantias adequadas de proteção (Brasil, 2000).

Segundo Faria e Pires (2012), gestão das unidades de conservação pode ser definida como tudo que é realizado dentro e fora das unidades com a intenção de se alcançar ou se aproximar dos objetivos de manejo das mesmas. Esse processo gerencial tem se tornado mais profissional, envolvendo a articulação de elementos técnicos, políticos e operacionais e requisitando capacitação em temáticas variadas. São apontadas como ações exercidas pelos gestores a administração dos recursos humanos e financeiros, elaboração de projetos, articulação política, proteção contra incêndios, presidência de conselhos consultivos, recepção de visitantes, elaboração de planos de manejo, monitoramento da qualidade ambiental, realização de palestras e eventos, dentre outras.

Todavia, as unidades de conservação enfrentam problemas, como falta de plano de manejo, de regularização fundiária, de recursos humanos qualificados, da proteção efetiva dos biomas, dentre outros. Pode ser apontada como causa principal de tais problemas a limitada destinação de recursos financeiros a essas áreas (Medeiros e Young, 2011; Geluda et al., 2014; Riva et al., 2014; Godoy e Leuzinger, 2015; Coutinho Júnior et al., 2016).

A criação de espaços naturais protegidos com a intenção de preservação da biodiversidade é declarada de extrema importância. Porém, a falta de recursos inviabiliza o alcance dos diversos objetivos das mesmas, trazendo como consequência a falta de infraestrutura, equipamentos, fiscalização, pessoal qualificado, o que acaba por facilitar usos inadequados, invasões, atividades ilegais e degradação ambiental (MMA, 2009; Medeiros e Young, 2011; Sales, 2012; Dias, 2013; Prates e Sousa, 2014; Riva et al., 2014; Geluda et al., 2015; Godoy e Leuzinger, 2015; Araújo et al., 2016).

Pode-se destacar que a eficiência da gestão financeira é fundamental para as unidades de conservação perpetuarem-se por muitos anos. Ainda, vale frisar que tanto as demandas como as fontes de recursos são dinâmicas e vinculadas a fatores políticos, econômicos, tecnológicos, culturais, locais e globais, tornando necessária a periódica avaliação e revisão dos métodos de gestão. Evidentemente, nem todos os problemas enfrentados na gestão das unidades de conservação são de ordem financeira, mas muitos dos outros obstáculos poderiam ser minimizados caso houvesse recursos adequados (Geluda et al., 2015).

A sustentabilidade financeira das unidades de conservação ainda pode ser considerada um dos maiores obstáculos à efetivação das mesmas no contexto atual (Dias, 2013; Geluda et al., 2014). Compreende-se por sustentabilidade financeira

\footnotetext{
a capacidade de se obter recursos estáveis e suficientes, longo prazo, para cobrir os custos necessários para um manejo eficiente, permitindo o alcance dos objetivos sociais, econômicos e ambientais das unidades de conservação (Geluda et al., 2014).
}

Considerando a conjuntura de adversidades relativas à disponibilização de recursos, as unidades de conservação necessitam desenvolver alternativas financeiras de alocação de recursos, a fim de manterem a preservação e conservação da biodiversidade (Lima, 2010; Lanna, 2012; Dias, 2013; Vedoveto et al., 2014; Godoy e Leuzinger, 2015).

\section{Uso público em unidades de conservação}

A criação das unidades de conservação alcança além da manutenção da biodiversidade, potencializando atividades ligadas à geração de renda, empregos e aumento da qualidade de vida. Aceita-se que existe o subaproveitamento do considerável potencial econômico de tais áreas, as quais precisam ser examinadas e analisadas além de 
seus objetivos precípuos de conservação, englobando aspectos de gestão, igualmente importantes (Dias, 2013; Geluda et al., 2014; Riva et al., 2014). 0 incentivo de atividades econômicas ambientalmente sustentáveis pode ser considerado como alternativa de financiamento, utilizando os recursos naturais de forma direta ou indireta e revertendo parte das receitas à conservação (Medeiros et al., 2011; Riva et al., 2014). 0 estabelecimento de parcerias entre setor público e o privado empresarial ou organizações da sociedade civil, a fim de aportar recursos e ferramentas direcionados à gestão das unidades de conservação, pode ajudar a transformar em realidade o potencial existente (Geluda et al., 2014; Riva et al., 2014; Araújo et al., 2016).

A utilização de concessões pode tornar-se mecanismo interessante na operação de unidades de conservação, desde que utilizada com base em plano de manejo estruturado, padrões de qualidade detalhados. A limitação do número de operadores de serviços, os quais em geral possuem exclusividade dentro da área, restringe os efeitos negativos sobre a biodiversidade de um possível uso intensivo dos recursos naturais (Gorini et al., 2006). Destaca-se que a exploração direta ou indireta dos recursos naturais, em quase todas as categorias de unidades de conservação no nível federal, depende de aprovação do ICMBio e está regulamentada na Instrução Normativa no 19/2011 (ICMBio, 2011) e também pela Instrução Normativa no 02/2017 (ICMBio, 2017), além de outras normas pertinentes.

Com o objetivo de gerar recursos, muitos países têm incentivado e permitido atividades econômicas sustentáveis nas unidades de conservação, como por exemplo a renda obtida pelo turismo e revertida para a conservação. A promoção do turismo sustentável faz sentido também porque, sendo realizada de maneira planejada, tem impacto ambiental relativamente baixo, comparando-se com outras atividades (Gorini et al., 2006; Riva et al., 2014). 0 turismo incentiva a preservação e conservação das unidades de conservação, além de apresentar a possibilidade de conciliar uso sustentável e a geração de receitas, pode ser revertida ao custeio dessas áreas (Bellinassi et al., 2011; Lanna, 2012; Riva et al., 2014; Godoy e Leuzinger, 2015).

A falta de infraestrutura adequada ao uso público, tal como centro de visitantes, trilhas, atrações recreativas, dentre outros, é problema comum às unidades de conservação brasileiras (Vedoveto et al., 2014). A visitação pública promove o envolvimento da população com a unidade de conservação, tornando-se ferramenta de valorização dos recursos naturais e socioculturais presentes na mesma. Além disso, proporciona retorno econômico importante, o qual subsidia financeiramente a alocação de verba do Estado direcionada à unidade, justificando tal investimento. Normalmente 0 investimento na visitação é somente de trilhas interpretativas, mas outros atrativos também podem contribuir para melhorias da mesma forma que a visitação, tais como atividades culturais, educativas e históricas (Bellinassi et al., 2011).

Realizar a associação do turismo com a biodiversidade pode gerar alternativas econômicas sustentáveis a muitas comunidades no Brasil. Incentivar o turismo em unidades de conservação aumenta a possibilidade de arrecadação de recursos para a manutenção das unidades, por meio de vendas de ingressos, taxas de realização de atividades recreativas e de uso de instalações, como campings, estacionamento e abrigos, cobrança por concessão de serviços, venda de mercadorias, dentre outros (MMA, 2009).

As unidades de conservação desempenham funções importantes para a humanidade, contudo tal fato não é reconhecido por todos. Desse modo, conhecer o valor de existência, valor de uso, de opção e não uso torna-se uma forma de fortalecer as unidades de conservação e perpetuar o fornecimento dos serviços ambientais (Aguilar, 2013). A exploração e concessão florestais podem ser apontadas como serviços ambientais, os quais podem proporcionar renda, empregos, investimentos externos e o retorno financeiro, contribuindo como fonte de receita (Lanna, 2012; Vedoveto et al., 2014; Godoy e Leuzinger, 2015). 
A presença de unidades de conservação constitui importante mecanismo destinado a garantir a oferta de água atual e futura, influenciando também no valor monetário da mesma. Nos mananciais e bacias hidrográficas com maior cobertura florestal, o custo do tratamento da água destinada ao abastecimento público diferencia-se do custo de tratamento em mananciais com baixa cobertura florestal, sendo o primeiro de menor valor (Medeiros e Young, 2011; Lanna, 2012; Godoy e Leuzinger, 2015).

\section{Materiais e métodos}

Na pesquisa referente a este estudo foram dados utilizados como técnicas de coleta de dados o levantamento bibliográfico e análise documental. Realizada no período compreendido entre os anos de 2016 e 2018.

Para a descrição dos aspectos relativos à conservação da biodiversidade em Minas Gerais, com informações sobre a gestão financeira das unidades de conservação, foi utilizado, além da pesquisa bibliográfica, a pesquisa documental.

A fim de realizar a análise dos dados coletados, foi utilizada técnica de análise de conteúdo (Bardin, 2011). Inicialmente foi realizada pré-análise, englobando leitura geral e organização, realizando a seleção do material a ser posteriormente analisado. Logo após, realizou-se a exploração do material, constituída de categorização e classificação temática básica de recortes de texto, facilitando a próxima etapa. Por último, foi realizada a interpretação, etapa destinada ao tratamento dos resultados, captando os conteúdos expostos e latentes, realizando inferências e análises reflexivas.

\section{Resultados}

\section{Minas Gerais: a gestão financeira das unidades de conservação}

O Estado de Minas Gerais é beneficiado com recursos naturais diversificados, tais como solos férteis, relevo e adequados, biodiversidade, jazidas minerais e bacias hidrográficas. De acordo com a WWF (2016), possui aproximadamente 6 milhões de hectares protegidos por meio de unidades de conservação dos três níveis da federação. Conforme Salvio (2017), existiam 307 áreas protegidas estaduais, divididas em 68 de proteção integral, 220 de uso sustentável e 19 áreas de proteção especial, no ano de 2017.

A indicação, implantação e gestão das unidades de conservação compete ao Instituto Estadual de Florestas (IEF), que tem como finalidade promover a preservação e conservação da fauna e flora e o desenvolvimento sustentável dos recursos naturais renováveis, assim como executar a política florestal do Estado. Além disso, apoiar atividades de regularização e fiscalização, bem como promover a educação ambiental e manter sistema de informação de conhecimentos técnicos relativos à biodiversidade. 0 IEF incorpora-se ao Sistema Estadual de Meio Ambiente e Recursos Hídricos (SISEMA) e à Secretaria Estado de Meio Ambiente e Desenvolvimento Sustentável (SEMAD) (Minas Gerais, 2011).

Em relação à gestão das unidades de conservação, o Instituto Estadual de Florestas é responsável pela administração de 91 unidades de conservação estaduais, dentre categorias diversas, 72 de proteção integral e 19 de uso sustentável, sendo dez áreas de proteção ambientais e três refúgios estaduais de vida silvestre. As unidades estão subordinadas à Diretoria de Unidades de Conservação (DIUC) e aos Escritórios Regionais de Florestas e Biodiversidade, diretamente ligados à Diretoria Geral do Instituto Estadual de Florestas. A primeira estrutura responde pela criação, implantação e gestão das unidades de conservação estaduais, e a segunda realiza apoio administrativo na execução das atividades planejadas. Sobre a efetividade de gestão, a das unidades de conservação estaduais pode ser considerada média (47\%). A efetividade das unidades de proteção integral também é média (48\%), já a efetividade das unidades de uso sustentável é baixa 
(38\%) (WWF, 2016).Entende-se que a consolidação do planejamento financeiro efetivo dos sistemas estaduais de unidades de conservação pode ser considerada elemento-chave da concretização das agendas de conservação da biodiversidade dos governos estaduais (Freitas e Camphora, 2009). De acordo com o documento Plano Mineiro de Desenvolvimento Integrado, é essencial que o Governo promova a expansão e criação de novas unidades de conservação, assim como o aprimoramento da estrutura das existentes, oferecendo apoio político e transparência na solução de conflitos de interesse (Minas Gerais, 2016a).

De acordo com estudo desenvolvido pelo TCE (2012), pode-se inferir que o quantitativo de investimentos realizados, a deficiência no planejamento dos recursos financeiros, humanos, materiais e tecnológicos e a precariedade na utilização dos instrumentos de arrecadação são fatores que ocasionam a vulnerabilidade da gestão das unidades de conservação mineiras, em especial as de proteção integral. Foi averiguado também que existem deficiências quanto à equidade da distribuição dos recursos, na transparência e no estabelecimento de critérios na alocação dos mesmos.

Segundo Freitas e Camphora (2009), um dos desafios da consolidação das unidades de conservação estaduais fundamenta-se em garantir mecanismos que proporcionem a efetiva sustentabilidade financeira das mesmas. De acordo com as autoras, foram encontrados em Minas Gerais algumas fontes financeiras principais, efetivas e potenciais voltadas à gestão do sistema estadual de unidades de conservação. São elas: orçamento público, recursos provenientes de compensação ambiental, cobrança de visitação em parques estaduais, concessões de uso público ou instalações de infraestrutura, pagamento por serviços ecossistêmicos, gestão compartilhada e sistemas de cogestão por OSCIP's.

De acordo a WWF (2016), o montante total de recursos financeiros executados no ano de 2014 nas unidades de conservação avaliadas no estudo foi cerca de 142 milhões de reais. Destaca-se que nesse estudo foram avaliadas $76 \%$ das unidades estaduais. A Tabela 1 apresenta os valores dispendidos em Minas Gerais, relativos ao orçamento público.

Tabela 1. Despesas e investimentos em unidades de conservação de Minas Gerais.

\begin{tabular}{|c|c|c|}
\hline Especificação & Valores (R\$) & Ano \\
\hline 1. Criação, gestão e implantação (despesas) & $25.131 .789,00$ & \multirow{6}{*}{2016} \\
\hline 1.1. Serviços de terceiros pessoa jurídica & $13.253 .794,00$ & \\
\hline 1.2. Serviços de terceiros pessoa física & $72.900,00$ & \\
\hline 1.3. Material de Consumo & $1.279 .895,00$ & \\
\hline 1.4. Locação de mão-de-obra & $1.868 .400,00$ & \\
\hline 1.5. Material permanente & $8.656 .800,00$ & \\
\hline 2. Regularização fundiária & $22.584 .000,00$ & 2016 \\
\hline 3. Investimentos & $84.451 .484,00$ & 2012 a 2015 \\
\hline 4. Estimativa criação, gestão e implantação & $106.229 .851,00$ & 2016 a 2019 \\
\hline
\end{tabular}

Fonte: Minas Gerais (2012), Minas Gerais (2016b) e Minas Gerais (2016c).

\section{Uso público, visitação e turismo: experiências bem-sucedidas e iniciativas governamentais}

0 aprimoramento e a capacitação institucional e administrativas voltados à gestão sustentável do uso público em unidades de conservação estaduais pode ser concebida como opção efetiva que assegure maior envolvimento da sociedade e de instituições públicas e privadas em estratégias de valorização da biodiversidade. A criação de sistemas 
de cobrança por visitação e a concessão de serviços de uso público propiciam à população o direito de acesso aos benefícios ambientais provenientes da conservação (Freitas e Camphora, 2009).

De acordo com estudo realizado pelo TCE (2012) com gerentes de unidades em Minas Gerais, a cobrança de taxa de visitação foi a mais citada dentre as possíveis fontes de arrecadação, seguida das parcerias e cobrança pela exploração da prática de esportes dentro das unidades. Foi averiguado no estudo, especificamente em relação aos parques, que tais unidades possuem demanda espontânea de visitação e atividades de lazer, mesmo não apresentando infraestrutura. A Figura 1 apresenta o montante estimado de recursos arrecadados decorrentes da visitação das unidades de conservação.

A valorização de mercado dos recursos hídricos, carbono e biodiversidade pode ser concebida como incentivo importante para a conservação, gerando renda e permitindo a distribuição equitativa de benefícios e custos relacionados à gestão da biodiversidade. No entanto, a base legal ainda não definiu regras relativas a tais pagamentos (Freitas e Camphora, 2009). Segundo estudo desenvolvido pelo TCE (2012), existe potencial de aproveitamento econômico em algumas unidades relativo aos recursos hídricos. Os órgãos públicos que utilizam os espaços protegidos como fonte de água para abastecimento da população poderiam remunerar tais unidades por essa retirada de recursos.

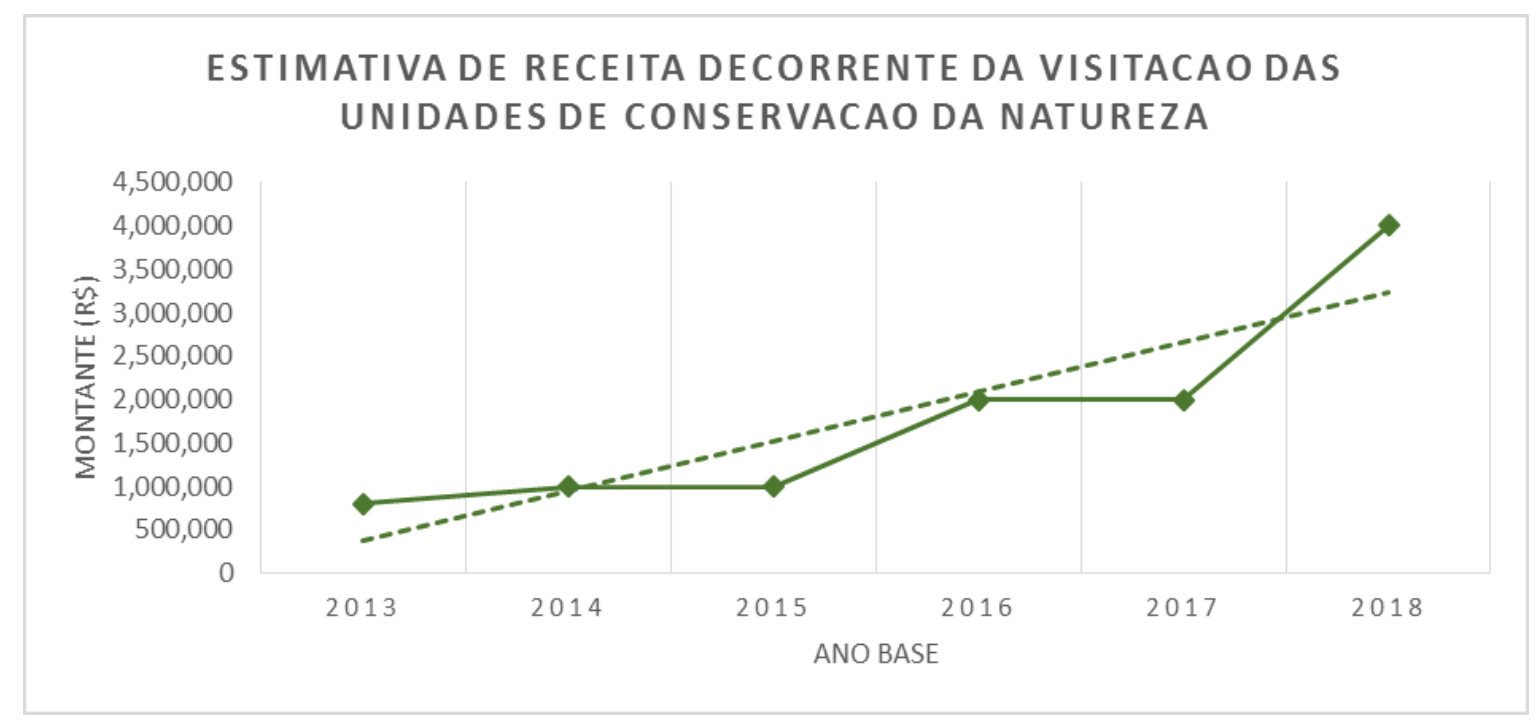

Figura 1. Gráfico da estimativa de arrecadação com visitação. Fonte: elaborada pela autora com base na pesquisa.

Miranda (2013) realizou estudo com objetivo de propor modelo de gestão integrada, a fim de conciliar promoção do turismo e conservação da biodiversidade. Foram entrevistados 38 gestores de parques em Minas Gerais, além de turistas, membros das comunidades e funcionários, abordando temas como conservação do espaço natural e prática de ecoturismo. Constatou-se que as práticas gerenciais não atendem satisfatoriamente às demandas do ecoturismo. Ainda, observou-se que o ecoturismo pode contribuir para o desenvolvimento local, além de desenvolver meios sustentáveis de gestão das unidades de conservação.

Santos (2010), com o objetivo de analisar o uso público em unidades de conservação, realizou estudo de caso no Parque Nacional do Iguaçu (PARNA do Iguaçu). 
Destacou-se a necessidade de um plano de uso público, voltado para os objetivos de manejo da unidade, bem como com a preocupação com a qualidade, eficiência e segurança. Concluiu-se que atividades ecoturísticas e de uso público ajudam a garantir o desenvolvimento destas unidades de conservação e permitem a melhoria da economia local. Ainda sobre o PARNA do Iguaçu, Gorini et al. (2006) desenvolveram estudo sobre possibilidades de exploração de novas tendências do turismo mundial pelas unidades de conservação brasileiras, em especial o ecoturismo, expondo casos mundiais de gestão e financiamento das unidades, indicando o caso como o mais bem-sucedido no Brasil.

$\mathrm{Na}$ região Amazônica denominada Terra do Meio, existem iniciativas de valorização da produção extrativista, por meio de mecanismos de geração de renda a partir da manutenção do modo tradicional de produção, constituindo instrumento de consolidação das áreas protegidas. Além disso, contribui para a permanência das populações tradicionais nas áreas, resultando em um controle e monitoramento maiores. No caso em questão, foram estabelecidos contratos de comercialização, respeitando os objetivos de criação das unidades e a legislação vigente, de dois produtos principais: a borracha e a copaíba (Gomes, 2012).

0 estudo desenvolvido pelo ICMBio, foram apresentados dois relatórios, em 2017 e 2018, de contribuições do turismo em unidades de conservação federais para a economia brasileira, provando, em números, que as áreas protegidas se tornaram vetores de desenvolvimento econômico (Souza et al., 2017; Souza e Simões, 2018). Demonstrou que, em dados de 2018, "a cada real investido no ICMBio produziu R\$ 7 em benefícios econômicos para o Brasil" (Souza e Simões, 2018). Ainda, o estudo informou que os impactos econômicos do turismo afetam diretamente a gestão das unidades de conservação e os empreendimentos turísticos. Os resultados demonstram a importância do turismo nas unidades de conservação e também nas regiões vizinhas e indicam que investimentos adicionais em uso público incentivarão o crescimento da visitação, gerando benefícios às comunidades e garantindo o desenvolvimento sustentável dos destinos turísticos (Souza et al., 2017; Souza e Simões, 2018).

O ICMBio conta com estratégias de implementação de visitação em unidades de conservação federais, por meio do programa Parcerias Ambientais Público-Privadas, com o objetivo de formular e fomentar alianças e parcerias público-privadas, visando o aproveitamento sustentável das potencialidades econômicas das unidades de conservação. Foi identificado que algumas áreas oferecem grandes oportunidades para gerar benefícios tanto econômicos como sociais, que podem produzir resultados financeiros e proporcional a sustentabilidade econômica e operacional das unidades de conservação (ICMBio, 2018).

O Guia Boas Práticas de Gestão aponta diversas oportunidades que podem ser implementadas, a fim de ampliar visitação em unidades de conservação e oferecer melhores experiências ao público. Algumas das boas práticas são parcerias com cooperados, regionalização das compras de produtos de pequenos agricultores, parcerias público privadas, parcerias institucionais, capacitação para a comunidade, interpretação ambiental, implantação de tecnologia de dados, sensoriamento remoto e visitação, pluriparticipação, gestão participativa, grupos de trabalho, comunicação e visibilidade, articulação e diálogo, grupos de visitantes, mobilização, cooperação técnica, turismo sustentável, visitação agendada, ordenamento participativo, parcerias locais, dentre outros (ICMBio, 2018a).

A proposta brasileira Travessias propõe trilhas de longo prazo em unidades de conservação, com o objetivo de promover recreação, conservação e arrecadação. Essa última é apontada pelo estudo como geradora de financiamento para a conservação, emprego e renda. A intenção de tal projeto é que as trilhas sirvam como conectores de paisagem entre unidades de conservação e outras áreas núcleo, tornando ferramenta de conservação um equipamento de recreação. A implementação começa pelas áreas protegidas, utilizando justamente o conjunto de travessias já existente, além das que 
pretendem ser implementadas em cada unidade de conservação federal (ICMBio, 2018b). Como parte integrante desse projeto, o ICMBio lançou um manual oficial de sinalização de trilhas, o qual permite a sinalização de forma simples e de baixo custo, acessível a qualquer unidade de conservação. Tem como objetivo as unidades de conservação federais, mas pode ser utilizado tanto na esfera municipal como estadual, além de trilhas não localizadas em áreas protegidas (ICMBio, 2018c).

\section{Discussão}

Foi observado que nas unidades de conservação de Minas Gerais existem deficiências quanto à equidade da distribuição dos recursos, na transparência e no estabelecimento de critérios na alocação dos mesmos, acarretando diversos problemas (TCE, 2012). Tal situação foi apontada por diversos autores, os quais indicam que a falta de recursos inviabiliza o alcance dos objetivos das unidades de conservação (MMA, 2009; Medeiros e Young, 2011; Sales, 2012; Dias, 2013; Geluda et al., 2014; Prates e Sousa, 2014; Riva et al., 2014; Geluda et al., 2015; Godoy e Leuzinger, 2015; Araújo et al., 2016; Coutinho Júnior et al., 2016).

Conforme estudo de Freitas e Camphora (2009), realizado em Minas Gerais, um dos desafios da consolidação das unidades de conservação estaduais fundamenta-se em garantir mecanismos que proporcionem a efetiva sustentabilidade financeira das mesmas, conforme apontam Dias (2013) e Geluda et al. (2014).

A cobrança da taxa visitação foi citada dentre as possíveis fontes de arrecadação, seguida de atividades recreativas nas unidades, de acordo com estudo realizado em Minas (TCE, 2012). 0 incentivo ao turismo sustentável encontra respaldo em vários autores, os quais indicam que a renda arrecadada pode vir a custear áreas protegidas (Bellinassi et al., 2011; Godoy e Leuzinger, 2015; Gorini et al., 2006; Lanna, 2012; Riva et al., 2014). Ainda, pelo gráfico apresentado no presente estudo, identifica-se um crescimento nos valores arrecadados com visitação, demonstrando que há um potencial a ser explorado e que as experiências estão sendo bem-sucedidas. Os resultados do estudo de contribuições do turismo em unidades de conservação federais para a economia demonstram a importância do turismo nas unidades (Souza et al., 2017; Souza e Simões, 2018). Gorini et al. (2006) e Santos (2010) apontam a experiência do PARNA do Iguaçu como demonstração de resultados positivos envolvendo uso público.

Foi constatado que existe potencial de aproveitamento econômicos de algumas unidades de Minas Gerais em relação aos recursos hídricos (TCE, 2012), conforme apontam Medeiros e Young (2011), Lanna (2012) e Godoy e Leuzinger (2015) a presença de unidades de conservação é mecanismo importante, que garante a oferta de água atual e futura. Miranda (2013), observou-se que as práticas gerenciais não atendem às demandas do turismo de forma satisfatória, conforme apontam Vedoveto et al. (2014), a falta de estrutura adequada ao uso público é realidade comum às unidades de conservação brasileiras. Bellinassi et al. (2011) apontam que, normalmente, o investimento na visitação é somente de trilhas interpretativas, porém o Guia Boas Práticas de Gestão aponta diversas oportunidades que podem ser implementadas, a fim de ampliar visitação em unidades de conservação e oferecer melhores experiências ao público (ICMBio, 2018a).

A exploração florestal pode proporcionar retorno financeiro, emprego e renda (Lanna, 2012; Vedoveto et al., 2014; Godoy e Leuzinger, 2015). Gomes (2012) aponta experiência positiva na Terra do Meio, onde a produção extrativista constitui instrumento de consolidação de áreas protegidas, geração de renda e permanência das populações tradicionais nessas áreas.

Sobre a importância das parcerias entre setor público e privado (Geluda et al., 2014; Riva et al., 2014; Araújo et al., 2016), o ICMBio está desenvolvendo o Programa Parcerias Ambientais Público-Privadas, justamente com o objetivo de gerar benefícios 
econômicos e sociais, produzir resultados financeiros e proporcionar a sustentabilidade econômica e operacional das unidades de conservação (ICMBio, 2018).

\section{Considerações finais}

Com base nos objetivos do estudo, chegou-se ao entendimento que, diante de dificuldades de disponibilização de recursos, as unidades devem ser analisadas também sob aspectos gerenciais e alternativas financeiras devem ser criadas. A taxa de visitação é considerada importante fonte de arrecadação, além das parcerias e cobrança pela exploração da prática de esportes dentro das unidades. Os artigos pesquisados reforçam a ideia de uso público nas unidades de conservação e demonstram a importância e pertinência do turismo sustentável. Foram observadas experiências positivas, tanto em Minas Gerais como no Brasil.

A pesquisa realizada aponta possibilidades de estudos futuros, porém com a inclusão de observações em campo, entrevistas com moradores, populações tradicionais, gestores, objetivando verificar se os esforços são continuados e apresentam consequências positivas na prática.

\section{Conflito de interesses}

A autora declara não haver conflitos de interesse.

\section{Referências}

Aguilar, A. S. Valorização econômica dos serviços ambientais em unidade de proteção integral. Estudo de caso do Monumento Natural da Mãe d'Água, Serra da Moeda, Brumadinho/MG: enfoque nos recursos hídricos. Florianópolis: Universidade Federal de Santa Catarina, 2013. (Dissertação de mestrado).

Araújo, E.; Barreto, P.; Baima, S.; Gomes, M. Quais os planos para proteger as unidades de conservação vulneráveis da Amazônia? Belém: Imazon, 2016. Disponível em: $<$ https://imazon.org.br/publicacoes/quais-os-planos-para-proteger-as-unidades-deconservacao-vulneraveis-da-amazonia/>. Acesso em: 20 dez. 2018.

Bardin, L. Análise de conteúdo. São Paulo: Edições 70, 2011.

Bellinassi, S.; Pavão, A.C.; Cardoso-Leite, E. Gestão e Uso Público de Unidades de Conservação: um olhar sobre os desafios e possibilidades. Revista Brasileira de Ecoturismo, v. 4, n. 2, p. 274-293, 2011. https://doi.org/10.34024/rbecotur.2011.v4.5918

Bensusan, N.; Prates, A. P. A diversidade cabe na unidade? Áreas protegidas no Brasil. Brasília: IEB, 2014.

Brasil. Lei no 9.985, de 18 de julho de 2000. Institui o Sistema Nacional de Unidades de Conservação da Natureza e dá outras providências. Disponível em: <http://www.planalto.gov.br/ccivil_03/leis/L9985.htm>. Acesso em: 07 fev. 2020.

Coutinho Júnior, J. A. de Oliveira, F. M., Gomes, P. I. J., Leite, P. D. P., Batista, S. P. O processo brasileiro de criação, implantação e manejo de unidades de conservação ambiental: o caso do Parque Estadual da Lapa Grande, em Montes Claros-MG. Anais do V Congresso em Desenvolvimento Social, 2016.

Dias, T. C. A. C. Unidades de conservação brasileiras: investimentos, custos de manejo e potencialidades econômicas. Macapá: Universidade Federal do Amapá, 2013. (Tese de doutorado). 
Faria, H. H.; Pires, A. S. Administração, manejo ou gestão de unidades de conservação? Periódico Eletrônico Fórum Ambiental da Alta Paulista, v. 8, n. 3, 2012. https://doi.org/10.17271/19800827832012276

Freitas, A.; Camphora, A. L. Contribuição dos estados brasileiros para a conservação da biodiversidade: diagnóstico financeiro das unidades de conservação estaduais. Brasília: The Natural Conservancy, 2009. Disponível em: <https://www.tnc.org.br/ content/dam/tnc/nature/en/documents/brasil/diagnosticofinanceiro-ucs.pdf $>$. Acesso em: 07 fev. 2019.

Geluda, L.; Serrão, M.; Lemos, R. Desafios para a sustentabilidade financeira das unidades de conservação no Brasil. In: Bensusan, N.; Prates, A. P. (Org.). A diversidade cabe na unidade? Áreas protegidas no Brasil. Brasília: IEB, 2014. p. 185-218.

Geluda, L.; Serrão, M.; Pinheiro, J. B.; Silva, M. A.; Ferrazoli, A.; Catapan, M. Ambiente financeiro das unidades de conservação estaduais do Acre: desafios e oportunidades. Rio de Janeiro: Funbio, 2015. Disponível em: <http://conservacao.mpambiental.org/wpcontent/uploads/2016/04/Ambiente-Financeiro-das-Unidades-de-Conservação-

Estaduais-do-Acre.pdf>. Acesso em 15 abr. 2019.

Godoy, L. R. C.; Leuzinger, M. D. O Financiamento do Sistema Nacional de Unidades de Conservação no Brasil. Características e tendências. Revista de Informação Legislativa, v. 52, n. 206, p. 223-243, 2015.

Gomes, P. C. Valorização da produção extrativista como instrumento de consolidação de áreas protegidas: a experiência da Terra do Meio. In: Fundo Vale (Ed.). Áreas protegidas. 1. ed. Rio de Janeiro: Fundo Vale, 2012. Disponível em: <https://imazon.org.br/ PDFimazon/Portugues/calha_norte/capitulos_de_livros/AreasProtegidasLivro.pdf > .

Acesso em: 15 abr. 2019.

Gorini, A. P. F.; Mendes, E. F.; Carvalho, D. M. P. Concessão de serviços e atrativos turísticos em áreas naturais protegidas: o caso do Parque Nacional do Iguaçu. BNDES Setorial, n. 24, p. 171-209, 2006.

ICMBio - Instituto Chico Mendes de Conservação da Biodiversidade. Parques do Brasil: visitar é proteger! Estratégias de implementação da visitação em unidades de conservação federais: prioridades de execução 2018-2020. Brasília: ICMBio, 2018. Disponível em: <http://www.icmbio.gov.br/portal/images/stories/comunicacao/publicacoes/ publicacoes-diversas/parques_do_brasil_estrategia_implementacao_ visitacao_2018_2020_ICMBio.pdf>. Acesso em: 25 out. 2019.

ICMBio - Instituto Chico Mendes de Conservação da Biodiversidade. Boas práticas na gestão de unidades de conservação. 3. ed. Brasília: ICMBio, 2018a. Disponível em: <http://www.icmbio.gov.br/portal/images/stories/comunicacao/publicacoes/publicacoe s-diversas/boas_praticas_na_gestao_de_ucs_edicao_3_2018.pdf>. Acesso em: 19 out. 2019.

ICMBio - Instituto Chico Mendes de Conservação da Biodiversidade. Travessias: uma aventura pelos parques nacionais do Brasil. 1. ed. Brasília: ICMBio, 2018b. Disponível em: <http://www.icmbio.gov.br/portal/images/stories/comunicacao/publicacoes/publicacoe s-diversas/travessias_uma_aventura_pelos_parques_nacionais_do_brasil.pdf $>$. Acesso em: 20 out. 2019.

ICMBio - Instituto Chico Mendes de Conservação da Biodiversidade. Manual de Sinalização de Trilhas. Brasília: ICMBio, 2018c. Disponível em: <http://www.icmbio.gov.br/portal/images/stories/comunicacao/publicacoes/publicacoe s-diversas/manual_de_sinalizacao_de_trilhas_ICMBio_2018.pdf>. Acesso em: 20 out. 2019. 
ICMBio - Instituto Chico Mendes de Conservação da Biodiversidade. Instrução Normativa no 02, de 30 de janeiro de 2017. Disponível em: <http://www.icmbio.gov.br/portal/ images/stories/portarias/intrucao_normativa_02_2017.pdf>. Acesso em: 20 out. 2019.

ICMBio - Instituto Chico Mendes de Conservação da Biodiversidade. Instrução Normativa no 19, de 16 de setembro de 2011. Disponível em: <http://www.icmbio.gov.br/portal/ images/stories/portarias/Instrucao_Normativa_19_de_16set2011.pdf >. Acesso em: 20 out. 2019.

Lanna, M. Finanças da conservação e captação de recursos. In: Cases, M. O. (Org.). Gestão de unidades de conservação: compartilhando uma experiência de capacitação. Brasília: WWF-Brasil, Ipê, 2012. p. 283-305.

Lima, A. F. A importância de uma gestão sustentável em unidades de conservação: o caso do Parque Ecológico Altamiro de Moura Pacheco - Goiás. Brasília: Universidade de Brasília, 2010. (Dissertação de mestrado).

Medeiros, R.; Young, C. E. F. Contribuição das unidades de conservação para a economia nacional: Relatório Final. Brasília: UNEP-WCMC, 2011. Disponível em: <https://www.mma.gov.br/estruturas/240/_arquivos/relatorio_final_contribuio_uc_para _a_economia_nacional_reduzido_240.pdf>. Acesso em: 11 nov. 2019.

Minas Gerais. Plano mineiro de desenvolvimento integrado 2016-2027. Belo Horizonte, 2016a. Disponível em: <http://www.planejamento.mg.gov.br>. Acesso em: 10 maio 2019.

Minas Gerais. Lei orçamentária anual 2016. Volume V. Secretaria de Planejamento e Gestão. Belo Horizonte, 2016b. Disponível em: <http://www.planejamento.mg.gov.br/ planejamento-e-orcamento/orcamento-do-estado-de-minas-gerais/orcamento-2016>.

Acesso em: 28 jun. 2019.

Minas Gerais. Plano plurianual de ação governamental 2012-2015. Volume II. Belo Horizonte, 2012. Disponível em: <http://www.almg.gov.br/acompanhe/planejamento_ orcamento_publico/ppag/>. Acesso em: 28 jun. 2019.

Minas Gerais. Plano plurianual de ação governamental 2016-2019. Volume II. Belo Horizonte, 2016c. Disponível em: <http://www.almg.gov.br/acompanhe/planejamento_ orcamento_publico/ppag/>. Acesso em: 28 jun. 2019.

Minas Gerais. Lei Delegada no 180, de 20 de janeiro de 2011. Dispõe sobre a estrutura orgânica da Administração Pública do Poder Executivo do Estado de Minas Gerais e dá outras providências. Disponível em: <https://www.almg.gov.br/consulte/legislacao/ completa/completa-nova-min.html?tipo=LDL\&num=180\&ano=2011>. Acesso em: 30 jun. 2019.

Miranda, A. B. L. Ecoturismo em unidades de conservação: proposta de gestão ecoeficiente e integrada. Lavras: Universidade Federal de Lavras, 2013. (Tese de doutorado).

MMA - Ministério do Meio Ambiente. Pilares para a sustentabilidade financeira do Sistema Nacional de Unidades de Conservação. Brasília: MMA, 2009. Disponível em: <https://www.mma.gov.br/estruturas/sbf2008_dap/_publicacao/149_publicacao161220 10113443.pdf>. Acesso em: 15 ago. 2019.

Muanis, M. M.; Serrão, M.; Geluda, L. Quanto custa uma unidade de conservação federal? Uma visão estratégica para o financiamento do Sistema Nacional de Unidades de Conservação (SNUC). Rio de Janeiro: Funbio, 2009. Disponível em: 
<https://www.funbio.org.br/wp-content/uploads/2017/07/QUANTO-CUSTA-UMAUNIDADE-DE-CONSERVACAO-FEDERAL.pdf >. Acesso em: 12 out. 2019.

Prates, A. P.; Sousa, N. O. M. Panorama geral das áreas protegidas no Brasil: desafios para o cumprimento da Meta 11 de Aichi. In: Bensusan, N.; Prates, A. P. (Org.). A diversidade cabe na unidade? Áreas protegidas no Brasil. Brasília: IEB, 2014. p. 83-119.

Quammen, D. Pacto com a Natureza. National Geographic, v. 17, n. 124, p. 58-81, 2016.

Riva, A. L.; Aidar, F.; Toledo, C.; Pages, M.; Laes, M.; Dutra, V. Unidades de conservação no Brasil: a contribuição do uso público para o desenvolvimento socioeconômico. São Paulo: Semeia, 2014. Disponível em: <http://www.terrabrasilis.org.br/ecotecadigital/index.php/ estantes/uso-publico-ecoturismo/2798-unidades-de-conservacao-no-brasil-acontribuicao-do-uso-publico-para-o-desenvolvimento-socioeconomico>. Acesso em: 28 set. 2019.

Sales, G. Desafios e perspectivas para gestores de unidades de conservação. In: Cases, M. O. (Org.). Gestão de unidades de conservação: compartilhando uma experiência de capacitação. Brasília: WWF-Brasil, Ipê, 2012. p. 307-328. Disponível em: <https://www.wwf.org.br/?32545/Gesto-de-unidades-de-conservao-compartilhandouma-experincia-de-capacitao>. Acesso em: 02 dez. 2019.

Santos, A. M. O ecoturismo, uso público e o Parque Nacional do Iguaçu. Periódico Eletrônico Fórum Ambiental da Alta Paulista, v. 6, n. 3, 2010. https://doi.org/ 10.17271/1980082763201082

Salvio, G. M. M. Áreas naturais protegidas e indicadores socioeconômicos: o desafio de conservação da natureza. Jundiaí: Paco Editorial, 2017.

Sousa, N. O. M.; Santos, F. R. P.; Salgado, M. A. S.; Araújo, F. F. S. (Org.). Dez anos de história: avanços e desafios do Sistema Nacional de Unidades de Conservação da Natureza. Brasília: MMA, 2011. Disponível em: <https://www.mma.gov.br/ estruturas/240/_publicacao/240_publicacao06072011055602.pdf>. Acesso em: 18 abr. 2019.

Souza, T. V. S. B.; Simões, H. B. Contribuições do turismo em unidades de conservação federais para a economia brasileira: efeitos dos gastos dos visitantes em 2017. Brasília: ICMBio, 2018. Disponível em: <http://www.icmbio.gov.br/portal/images/stories/ comunicacao/publicacoes/publicacoes-diversas/contribuicoes_economicas_turismo_ 2018.pdf>. Acesso em: 25 out. 2019.

Souza, T. V. S. B.; Thapa, B.; Rodrigues, C. G. O.; Imori, D. Contribuições do turismo em unidades de conservação federais para a economia brasileira: efeitos dos gastos dos visitantes em 2015. Brasília: ICMBio, 2017. Disponível em: <http://www.icmbio.gov.br/ portal/images/stories/comunicacao/publicacoes/publicacoes-diversas/contribuicao_ do_turismo_em_uc_federais_para_a_economia_brasileira.pdf.pdf>. Acesso em: 25 out. 2019.

TCE-MG - Tribunal de Contas do Estado de Minas Gerais. Relatório final de auditoria operacional: meio ambiente - unidades de conservação de proteção integral. Belo Horizonte: TCU-MG, 2012. Disponível em: <https://www.tce.mg.gov.br/IMG/Auditoria Operacional/RELATÓRIO FINAL UCPIs.pdf>. Acesso em: 09 fev. 2020. 
Vedoveto, M.; Ortega, V.; Pereira, J.; Veríssimo, A. Desafios para a consolidação das unidades de conservação estaduais do Pará: financiamento e gestão. Belém: Imazon, 2014. Disponível em: <https://imazon.org.br/PDFimazon/Portugues/livros/Desafios para consolidacao de 0UCs no PA.pdf>. Acesso em: 09 fev. 2020.

WWF Brasil; ICMBio. Implementação da Avaliação Rápida e Priorização da Gestão de Unidades de Conservação (RAPPAM) em unidades de conservação estaduais de Minas Gerais. Brasília: WWF Brasil; ICMBio, 2016. Disponível em: <http://d3nehc6yl9qzo4.cloudfront.net/img/original/rappam_mg_web.pdf>. Acesso em: 23 nov. 2019. 